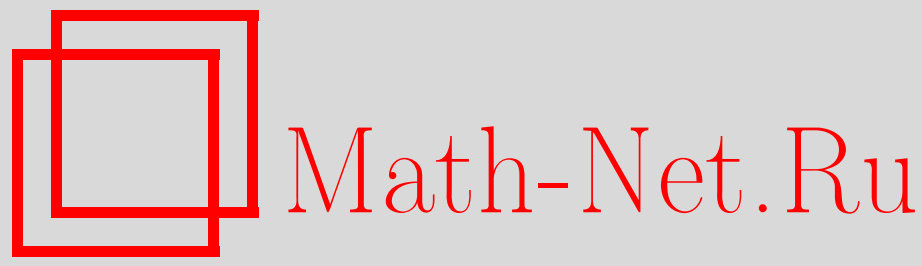

K. А. Попков, О тестах замыкания для контактных схем, Дискрет. матем., 2016, том 28, выпуск 1, 87-100

DOI: https://doi.org/10.4213/dm1359

Использование Общероссийского математического портала Math-Net.Ru подразумевает, что вы прочитали и согласны с пользовательским соглашением http://www.mathnet.ru/rus/agreement

Параметры загрузки:

IP: 54.210 .77 .194

26 апреля 2023 г., 13:09:23

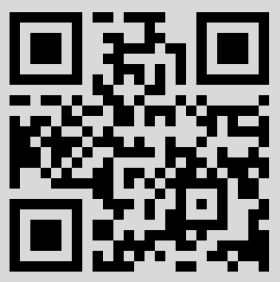




\title{
О тестах замыкания для контактных схем
}

\author{
(c) 2016 г. K. A. Попков*
}

Рассматривается задача синтеза двухполюсных контактных схем, реализующих булевы функции от $n$ переменных и допускающих короткие проверяющие и диагностические тесты относительно замыканий контактов. Установлено, что почти все булевы функции от $n$ переменных реализуемы неизбыточными двухполюсными контактными схемами, допускающими единичные проверяющие, полные проверяющие и единичные диагностические тесты константной длины. Доказано также, что:

1) любую булеву функцию $f\left(x_{1}, \ldots, x_{n}\right)$ можно реализовать неизбыточной двухполюсной контактной схемой, содержащей не более одной входной переменной, отличной от переменных $x_{1}, \ldots, x_{n}$, и допускающей единичный и полный проверяющий тесты длины не более $2 n$;

2) любую булеву функцию $f\left(x_{1}, \ldots, x_{n}\right)$ можно реализовать неизбыточной двухполюсной контактной схемой, содержащей не более двух входных переменных, отличных от переменных $x_{1}, \ldots, x_{n}$, и допускающей единичный диагностический тест длины не более $4 n$.

Работа выполнена при поддержке РФФИ, проект № 14-01-00598 ("Вопросы синтеза, сложности и контроля управляющих систем) и программы фундаментальных исследований ОМН РАН "Алгебраические и комбинаторные методы математической кибернетики и информационные системы нового поколения"(проект "Задачи оптимального синтеза управляющих систем").

Ключевые слова: контактная схема, замыкание контакта, проверяющий тест, диагностический тест

\section{1. Введение}

В статье рассматривается задача синтеза легкотестируемых двухполюсных контактных схем [1], реализующих заданные булевы функции. (Слово "двухполюсная" в дальнейшем будем опускать.) Логический подход к тестированию контактных схем предложен С. В. Яблонским и И. А. Чегис в [2]. Представим, что имеется контактная схема $S$, реализующая булеву функцию $f\left(x_{1}, \ldots, x_{n}\right)$. Под воздействием некоторого источника неисправностей один или несколько контактов схемы $S$ могут перейти в неисправное состояние. (В качестве неисправностей контактов обычно рассматриваются их обрывы и замыкания. При обрыве контакта проводимость между его концами становится тождественно нулевой, а при замыкании - тождественно единичной.) В результате схема $S$ вместо исходной функции $f\left(x_{1}, \ldots, x_{n}\right)$

*Место работы: ИПМ им. М. В. Келдыша РАН, e-mail: kirill-formulist@mail.ru 
будет реализовывать некоторую булеву функцию $g\left(x_{1}, \ldots, x_{n}\right)$, вообще говоря, отличную от $f$. Все такие функции $g\left(x_{1}, \ldots, x_{n}\right)$, получающиеся при всевозможных допустимых для рассматриваемой задачи неисправностях контактов схемы $S$, называются функциями неисправности данной схемы.

Введём следующие определения ([3-5]). Проверяющим тестом для схемы $S$ называется такое множество $T$ наборов значений переменных $x_{1}, \ldots, x_{n}$, что для любой отличной от $f\left(x_{1}, \ldots, x_{n}\right)$ функции неисправности схемы $S$ в $T$ найдётся набор $\tilde{\sigma}$, на котором $f(\tilde{\sigma}) \neq g(\tilde{\sigma})$. Диагностическим тестом для схемы $S$ называется такое множество $T$ наборов значений переменных $x_{1}, \ldots, x_{n}$, что $T$ является проверяющим тестом и, кроме того, для любых двух различных функций неисправности $g_{1}\left(x_{1}, \ldots, x_{n}\right)$ и $g_{2}\left(x_{1}, \ldots, x_{n}\right)$ схемы $S$ в $T$ найдётся набор $\tilde{\sigma}$, на котором $g_{1}(\tilde{\sigma}) \neq g_{2}(\tilde{\sigma})$. Число наборов в $T$ называется длиной теста. В качестве тривиального диагностического (и проверяющего) теста длины $2^{n}$ для схемы $S$ всегда можно взять множество $T$, состоящее из всех двоичных наборов длины $n$. Тест называется полным, если он применим к схемам с любым числом неисправных контактов, и единичным, если он применим к схемам, в которых может быть неисправен только один контакт. Единичные тесты обычно рассматривают для неизбыточных схем [5], т.е. для таких схем, в которых любая допустимая неисправность любого одного контакта приводит к функции неисправности, отличной от реализуемой данной схемой исходной функции (такие функции неисправности называют нетривиальными). Если в схеме допускаются только обрывы контактов (или только их замыкания), то говорят о тестах размыкания (соответственно о тестах замыкания).

Пусть зафиксирован вид неисправностей контактов и $T$ - единичный проверяющий тест для некоторой контактной схемы $S$. Пусть:

$D_{s, \operatorname{detect}}(T)$ - длина теста $T$,

$D_{s, \text { detect }}(S)=\min D_{s, \text { detect }}(T)$, где минимум берётся по всем тестам $T$ для контактной схемы $S$,

$D_{s, \text { detect }}(f)=\min D_{s, \text { detect }}(S)$, где минимум берётся по всем неизбыточным контактным схемам $S$, реализующим функцию $f$,

$D_{s, \text { detect }}(n)=\max D_{s, \text { detect }}(f)$, где максимум берётся по всем булевым функциям $f$ от $n$ переменных.

Функция $D_{s, \text { detect }}(n)$ называется функиией Шеннона длины единичного проверяющего теста. По аналогии с функциями $D_{s, \text { detect }}$ можно ввести функции $D_{c, \text { detect }}$, $D_{s, \text { diagn }}$ и $D_{c, \text { diagn }}$ для соответственно полного проверяющего, единичного диагностического и полного диагностического тестов, зависящие от $T$, от $S$, от $f$ и от $n$ (в определениях функций $D_{c, \text { detect }}(f)$ и $D_{c, \text { diagn }}(f)$ не требуется предполагать неизбыточность схем). Так, например, $D_{c, \text { diagn }}(n)-$ функция Шеннона длины полного диагностического теста.

Всюду будем предполагать, что контактная схема, реализующая булеву функцию $f\left(x_{1}, \ldots, x_{n}\right)$, реализует также и все булевы функции, получающиеся из $f\left(x_{1}, \ldots, x_{n}\right)$ изъятием всех или части фиктивных переменных этой функции. Это предположение согласуется с общепринятым положением теории булевых функций о том, что две булевы функции, получающиеся одна из другой при помощи операций добавления и изъятия фиктивных переменных, считаются равными (см., 
например, [6, с. 12]). Будем говорить, что контактная схема содержит $k$ фиктивных входных переменных и реализует функцию $f\left(x_{1}, \ldots, x_{n}\right)$ (где $\left.k \geqslant 0\right)$, если данная схема содержит $k$ различных входных переменных, отличных от переменных $x_{1}, \ldots, x_{n}$, и реализует булеву функцию, не зависящую существенно от этих $k$ переменных и равную функции $f\left(x_{1}, \ldots, x_{n}\right)$. Например, схема, изображенная на рис. 1 , содержит одну фиктивную входную переменную $y$ и реализует функцию $x_{1} x_{2}$. Будем говорить, что контактная схема реализует функцию $f\left(x_{1}, \ldots, x_{n}\right)$ (без указания числа фиктивных входных переменных), если это число равно нулю. Условимся считать, что наборы из любого теста для схемы, содержащей $k$ фиктивных входных переменных и реализующей функцию $f\left(x_{1}, \ldots, x_{n}\right)$, имеют длину $n+k$ (по общему числу переменных $x_{1}, \ldots, x_{n}$ и фиктивных входных переменных схемы). Такое предположение сделано в [7], где рассматриваются, в частности, контактные схемы, содержащие входные переменные $x_{0}, x_{1}, \ldots, x_{n}$ и реализующие функцию $f\left(x_{1}, \ldots, x_{n}\right)$, и тесты для этих схем, содержащие наборы длины $n+1$.

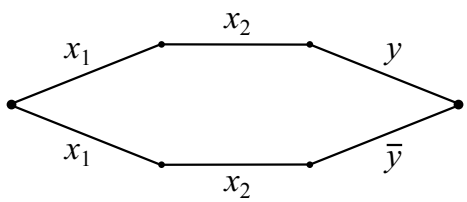

Рис. 1.

Будем говорить, что некоторое свойство выполняется для почти всех булевых функицй от $n$ переменных (или для почти каждой булевой функици от $n$ переменных), если отношение числа булевых функций от $n$ переменных, для которых это свойство не выполняется, к числу всех булевых функций от $n$ переменных (т.е. к $2^{2^{n}}$ ) стремится к нулю при $n \rightarrow \infty$.

Перечислим основные результаты, касающиеся тестирования контактных схем. Для случая, когда допускаются как обрывы, так и замыкания контактов, установлено, что функция $D_{s, \text { diagn }}(n)$ асимптотически не превосходит $\frac{2^{n+1}}{n}$ (см. $[5$, с. 113, теорема 9]); Х. А. Мадатян в [8] нашёл точное значение функции Шеннона длины полного диагностического теста: $D_{c, \text { diagn }}(n)=2^{n} ;$ Н. П. Редькин в [9] получил оценку $D_{c, \text { detect }}(n) \leqslant \frac{15}{16} \cdot 2^{n}$. Следующие оценки относятся к случаям, когда допускаются только обрывы контактов или только их замыкания. С использованием соображений, аналогичных использованным в [5] при доказательстве теоремы 9 на с. 113, в обоих случаях можно получить асимптотическую оценку $D_{s, \operatorname{diagn}}(n) \lesssim \frac{2^{n}}{n}$. Н. П. Редькиным в [10] для единичных проверяющих тестов размыкания и замыкания получены оценки соответственно $D_{s, \text { detect }}(n) \leqslant 2^{\left\lfloor\frac{n}{2}\right\rfloor}+2^{\left\lceil\frac{n}{2}\right\rceil}$ и $D_{s, \text { detect }}(n) \lesssim 2^{\frac{n}{1+\frac{1}{2 \log _{2} n}}+\frac{5}{2}}$. Отметим, что при доказательстве указанных результатов для булевых функций $f\left(x_{1}, \ldots, x_{n}\right)$ рассматриваются только реализующие их схемы без фиктивных входных переменных. Д. С. Романов в [7], в частности, установил, что для почти всех булевых функций $f$ от $n$ переменных справедлива оценка $D_{s, \text { detect }}(f) \leqslant 2 n+2$ длины единичного проверяющего теста замыкания, и что верна аналогичная оценка для единичного проверяющего теста размыкания, причём для 
доказательства обоих этих фактов использовались контактные схемы, содержащие одну фиктивную входную переменную $x_{0}$ и реализующие функцию $f\left(x_{1}, \ldots, x_{n}\right)$.

Вышеупомянутые верхние оценки для функций $D_{s, \text { detect }}(n), D_{s, \text { detect }}(f)$ в $[7,10]$ справедливы и для соответствующих функций $D_{c, \text { detect }}(n), D_{c, \text { detect }}(f)$, поскольку любой единичный проверяющий тест размыкания (замыкания) для неизбыточной контактной схемы является также и полным проверяющим тестом размыкания (замыкания) для этой схемы (см., например, [5, с. 147-148]). Используя это обстоятельство, из теорем 1, 2 и следствия 1 данной работы, которые будут сформулированы и доказаны ниже, можно получить аналогичные им результаты для величины $D_{c, \text { detect }}(f)$ и полных проверяющих тестов. Отдельно это оговаривать не будем.

В качестве неисправностей контактов будем рассматривать только их замыкания.

Множество $M$ двоичных наборов длины $n$, где $n \geqslant 2$, назовём покрывающим для булевой функции $f\left(x_{1}, \ldots, x_{n}\right)$, если на всех наборах из $M$ функция $f$ обращается в нуль и существует такой индекс $i \in\{1, \ldots, n\}$, что для любого индекса $j \in\{1, \ldots, n\}$, отличного от $i$, и для любых булевых констант $\alpha, \beta$ в $M$ найдётся набор, $i$-я компонента которого равна $\alpha$, а $j$-я $-\beta$.

Отметим, что покрывающее множество всегда непусто.

Пример 1. Если булева функция $f\left(x_{1}, \ldots, x_{n}\right)$ обращается в нуль на наборах $\left(0, \sigma_{2}, \ldots, \sigma_{n}\right),\left(0, \overline{\sigma_{2}}, \ldots, \overline{\sigma_{n}}\right),\left(1, \delta_{2}, \ldots, \delta_{n}\right),\left(1, \overline{\delta_{2}}, \ldots, \overline{\delta_{n}}\right)$, то в качестве покрывающего для $f$ множества можно взять множество, состоящее из этих четырех наборов (при этом $i=1$ ).

Пример 2. Пусть $M_{0}=\{(0,0,0,0),(0,0,1,0),(0,1,0,0),(0,1,0,1),(1,0,0,0)$, $(1,0,1,1)\}$ - множество всех нулевых наборов булевой функции $f\left(x_{1}, x_{2}, x_{3}, x_{4}\right)$. Тогда в качестве покрывающего для $f$ множества можно взять само $M_{0}$ или множество $\{(0,0,1,0),(0,1,0,0),(0,1,0,1),(1,0,0,0),(1,0,1,1)\}$ (при этом $i=4)$.

Пример 3. Пусть $M_{0}=\{(0,0,0,0),(0,1,0,0),(0,1,0,1),(1,0,0,0),(1,0,1,1)\}$ - множество всех нулевых наборов булевой функции $f\left(x_{1}, x_{2}, x_{3}, x_{4}\right)$. Тогда нетрудно убедиться, что покрывающего для $f$ множества не существует (для этого достаточно отдельно рассмотреть случаи $i=1,2,3,4)$.

Утверждение 1. Для почти каждой булевой функции от $n$ переменных существуют по крайней мере два непересекающихся покрывающих множества, каждое из которых содержит четыре набора.

Доказательство. Пусть $F_{n}-$ множество всех булевых функций от $n$ переменных, для каждой из которых указанное в условии утверждения свойство не выполняется; $f$ - произвольная функция из $F_{n}$. Разобьём все двоичные наборы длины $n$ на $2^{n-1}$ пар наборов, совпадающих только в первом разряде (ясно, что это можно сделать). Множество таких пар наборов с нулем в первом разряде обозначим через $A_{0}$, а с единицей в первом разряде - через $A_{1}$. Покажем, что функция $f$ удовлетворяет по крайней мере одному из следующих условий:

а) существует не более одной такой пары наборов из $A_{0}$, что функция $f$ равна нулю на обоих наборах из этой пары;

б) существует не более одной такой пары наборов из $A_{1}$, что функция $f$ равна нулю на обоих наборах из этой пары. 
Предположим, что это не так. Тогда существуют такие пары наборов ( $\tilde{\sigma}_{0}$, $\left.\tilde{\delta}_{0}\right) \in A_{0},\left(\tilde{\sigma}_{0}^{\prime}, \tilde{\delta}_{0}^{\prime}\right) \in A_{0},\left(\tilde{\sigma}_{1}, \tilde{\delta}_{1}\right) \in A_{1},\left(\tilde{\sigma}_{1}^{\prime}, \tilde{\delta}_{1}^{\prime}\right) \in A_{1}$, что все наборы $\tilde{\sigma}_{0}, \tilde{\delta}_{0}, \tilde{\sigma}_{0}^{\prime}, \tilde{\delta}_{0}^{\prime}$, $\tilde{\sigma}_{1}, \tilde{\delta}_{1}, \tilde{\sigma}_{1}^{\prime}, \tilde{\delta}_{1}^{\prime}$ попарно различны и являются нулевыми для функции $f$. В таком случае множества $M=\left\{\tilde{\sigma}_{0}, \tilde{\delta}_{0}, \tilde{\sigma}_{1}, \tilde{\delta}_{1}\right\}$ и $M^{\prime}=\left\{\tilde{\sigma}_{0}^{\prime}, \tilde{\delta}_{0}^{\prime}, \tilde{\sigma}_{1}^{\prime}, \tilde{\delta}_{1}^{\prime}\right\}$ будут покрывающими для $f$. Действительно, для любого $j \in\{2, \ldots, n\}$ и для любых булевых констант $\alpha, \beta$ первая компонента каждого из наборов $\tilde{\sigma}_{\alpha}, \tilde{\delta}_{\alpha}$ равна $\alpha$, а $j$-е компоненты этих наборов различаются (это следует из определения множеств $A_{0}, A_{1}$ ) и хотя бы одна из них равна $\beta$, откуда следует, что $M$ является покрывающим множеством для $f($ при $i=1$ ). Аналогичные рассуждения можно провести и для $M^{\prime}$. Каждое из этих множеств содержит четыре набора, и $M \cap M^{\prime}=\varnothing$, т.е. для функции $f$ указанное в условии утверждения 1 свойство выполняется, но это противоречит тому, что $f \in F_{n}$. Таким образом, любая функция $f$ из $F_{n}$ удовлетворяет хотя бы одному из условий а), б).

Найдём общее число булевых функций $f$ от $n$ переменных, удовлетворяющих условию а). На каждой паре наборов из $A_{0}$, кроме, быть может, одной, функция $f$ может принимать одну из трёх возможных пар значений - $(0,1),(1,0)$ или $(1,1)$. Всего в $A_{0}$ содержится $2^{n-2}$ пар наборов, а $2^{n-1}$ наборов не принадлежат никакой паре из $A_{0}$. Отсюда следует, что число булевых функций, принимающих на всех парах наборов из $A_{0}$ одну из трёх пар значений - $(0,1),(1,0)$ или $(1,1)$, равно $3^{2^{n-2}} \cdot 2^{2^{n-1}}$, а также что число булевых функций, принимающих на всех парах наборов из $A_{0}$, кроме одной, одну из этих трёх пар значений, а на одной паре наборов из $A_{0}$ - пару значений $(0,0)$, равно $C_{2^{n-2}}^{2^{n-2}-1} \cdot 3^{2^{n-2}-1} \cdot 2^{2^{n-1}}$. Таким образом, общее число булевых функций от $n$ переменных, удовлетворяющих условию а), равно $3^{2^{n-2}} \cdot 2^{2^{n-1}}+C_{2^{n-2}}^{2^{n-2}-1} \cdot 3^{2^{n-2}-1} \cdot 2^{2^{n-1}}=3^{2^{n-2}-1} \cdot 2^{2^{n-1}}\left(3+2^{n-2}\right)$. Аналогично общее число булевых функций $f$ от $n$ переменных, удовлетворяющих условию б), равно $3^{2^{n-2}-1} \cdot 2^{2^{n-1}}\left(3+2^{n-2}\right)$. Следовательно, число функций $f$ от $n$ переменных, удовлетворяющих хотя бы одному из условий а), б), не превосходит $2 \cdot 3^{2^{n-2}-1} \cdot 2^{2^{n-1}}\left(3+2^{n-2}\right)=3^{2^{n-2}-1} \cdot 2^{2^{n-1}+1}\left(3+2^{n-2}\right)$. Значит, $\left|F_{n}\right| \leqslant 3^{2^{n-2}-1} \cdot 2^{2^{n-1}+1}\left(3+2^{n-2}\right)$. Но тогда при $n \geqslant 4$ имеем

$$
\begin{aligned}
& \frac{\left|F_{n}\right|}{2^{2^{n}}} \leqslant \frac{3^{2^{n-2}-1} \cdot 2^{2^{n-1}+1}\left(3+2^{n-2}\right)}{2^{2^{n}}}=\frac{3^{2^{n-2}}\left(3+2^{n-2}\right)}{3 \cdot 2^{2^{n-1}-1}} \\
& \leqslant \frac{3^{2^{n-2}} \cdot 2^{n-1}}{3 \cdot 2^{2^{n-1}-1}}=\frac{2^{2^{n-2} \log _{2} 3}}{3 \cdot 2^{2^{n-1}-n}}=\frac{1}{3 \cdot 2^{2^{n-2}\left(2-\log _{2} 3\right)-n}} \rightarrow 0 \quad(n \rightarrow \infty),
\end{aligned}
$$

откуда следует справедливость утверждения 1.

Далее для краткости всюду вместо "замыкающий (размыкающий) контакт, отвечающий переменной $x_{j}{ }^{\prime}, j=1, \ldots, n$, будем говорить "контакт $x_{j}$ (соответственно контакт $\left.\overline{x_{j}}\right)$ ".

\section{2. Единичные проверяющие тесты замыкания}

Теорема 1. Пусть для булевой функиии $f\left(x_{1}, \ldots, x_{n}\right)$, где $n \geqslant 3$, существует покрывающее множество $M$ из $m$ наборов. Тогда $D_{s, \text { detect }}(f) \leqslant m$, причём существует такая неизбыточная контактная схема, реализующая функцию $f\left(x_{1}, \ldots, x_{n}\right)$, что множество $M$ для неё является единичным проверяющим тестом замыкания. 
Из теоремы 1 и утверждения 1 вытекает

Следствие 1. Для почти всех булевых функций $f$ om $n$ переменных справедливо неравенство $D_{\text {s, detect }}(f) \leqslant 4$.

Доказательство (теоремы 1). В определении покрывающего множества $M$ для функции $f\left(x_{1}, \ldots, x_{n}\right)$ будем без ограничения общности считать, что $i=1$ (в противном случае можно соответствующим образом переименовать отвечающие контактам переменные в контактной схеме, которая будет построена далее). Тогда на всех наборах из $M$ функция $f$ обращается в нуль и для любого индекса $j \in\{2, \ldots, n\}$, отличного от $i$, и для любых булевых констант $\alpha, \beta$ найдётся набор $\tilde{\pi}_{j, \alpha, \beta}$, первая компонента которого равна $\alpha$, а $j$-я $-\beta$. Если $f \equiv 0$, то функцию $f$ можно реализовать схемой, не содержащей ни одного контакта; такая схема неизбыточна и любое множество двоичных наборов длины $n$, в том числе и $M$, для неё является единичным проверяющим тестом, что и требовалось доказать. Далее будем считать, что функция $f$ отлична от тождественного нуля. Для каждого единичного для $f$ набора $\tilde{\sigma}_{k}=\left(\sigma_{k, 1}, \ldots, \sigma_{k, n}\right)$ построим контактную схему $S_{k}$ следующим образом. Вначале построим цепь $C_{k}$ из контактов $x_{1}^{\sigma_{k, 1}}, x_{2}^{\sigma_{k, 2}}, x_{1}^{\sigma_{k, 1}}, x_{3}^{\sigma_{k, 3}}, \ldots, x_{1}^{\sigma_{k, 1}}, x_{n}^{\sigma_{k, n}}$, соединённых в указанном порядке. Пусть $A, a_{1}, b_{1}, a_{2}, b_{2}, \ldots, a_{n-2}, b_{n-2}, a_{n-1}, B-$ вершины этой цепи при движении от первого контакта $x_{1}^{\sigma_{k, 1}}$ к контакту $x_{n}^{\sigma_{k, n}}$. Теперь соединим вершину $A$ с каждой вершиной $a_{s}, s=1, \ldots, n-1$, контактом $x_{s+1}^{\overline{\sigma_{k, s+1}}}$, а вершину $B-$ с каждой вершиной $b_{s}, s=1, \ldots, n-2$, контактом $x_{1}^{\overline{\sigma_{k, 1}}}$. Полученную контактную схему с полюсами $A$ и $B$ обозначим через $S_{k}$ (вид этой схемы при $n=5$ показан на рис. 2). Пусть $S$ - контактная схема, представляющая собой параллельное соединение всех построенных схем $S_{k}$. Легко видеть, что в каждой схеме $S_{k}$ лишь одна несамопересекающаяся цепь между её полюсами, а именно $C_{k}$, обладает ненулевой проводимостью и что функция, реализуемая схемой $S_{k}$, равна $x_{1}^{\sigma_{k, 1}} \& x_{2}^{\sigma_{k, 2}} \& \ldots \& x_{n}^{\sigma_{k, n}}$. Схема $S$ реализует дизъюнкцию всех таких функций для всех единичных наборов $\tilde{\sigma}_{k}$ функции $f$, т.е. совершенную дизъюнктивную нормальную форму для $f$, а значит, саму $f$. Докажем, что схема $S$ неизбыточна и множество $M$ для неё является единичным проверяющим тестом. Так как все наборы из $M$ нулевые для функции $f$, достаточно доказать, что при неисправности, т.е. при замыкании, любого одного контакта в схеме $S$ найдётся набор из $M$, на котором функция, реализуемая полученной схемой, равна единице. Предположим, что неисправен некоторый контакт в некоторой подсхеме $S_{k}$ схемы $S$. Возможны семь случаев.

1. Неисправен контакт $x_{1}^{\sigma_{k, 1}}$, соединяющий вершины $A$ и $a_{1}$ схемы $S_{k}$. Тогда на наборе $\tilde{\pi}_{2, \overline{\sigma_{k, 1}}}, \sigma_{k, 2}$ цепь $A-x_{1}^{\sigma_{k, 1}}-a_{1}-x_{2}^{\sigma_{k, 2}}-b_{1}-x_{1}^{\overline{\sigma_{k, 1}}}-B$ будет проводящей и схема $S_{k}$, а значит, и $S$, выдаст единицу.

2. Неисправен контакт $x_{1}^{\sigma_{k, 1}}$, соединяющий вершины $b_{s-1}$ и $a_{s}$ схемы $S_{k}$ для некоторого $s \geqslant 2$. Тогда на наборе $\tilde{\pi}_{s+1, \overline{\sigma_{k, 1}}}, \overline{\sigma_{k, s+1}}$ цепь $A-x_{s+1}^{\overline{\sigma_{k, s+1}}}-a_{s}-x_{1}^{\sigma_{k, 1}}-b_{s-1}-$ $x_{1}^{\overline{\sigma_{k, 1}}}-B$ будет проводящей и схема $S_{k}$, а значит, и $S$, выдаст единицу.

3. Неисправен контакт $x_{1}^{\overline{\sigma_{k, 1}}}$, соединяющий вершины $b_{s-1}$ и $B$ схемы $S_{k}$ для некоторого $s \geqslant 2$. Тогда на наборе $\tilde{\pi}_{s+1, \sigma_{k, 1}, \overline{\sigma_{k, s+1}}}$ цепь $A-x_{s+1}^{\overline{\sigma_{k, s+1}}}-a_{s}-x_{1}^{\sigma_{k, 1}}-b_{s-1}-$ $x_{1}^{\overline{\sigma_{k, 1}}}-B$ будет проводящей и схема $S_{k}$, а значит, и $S$, выдаст единицу. 


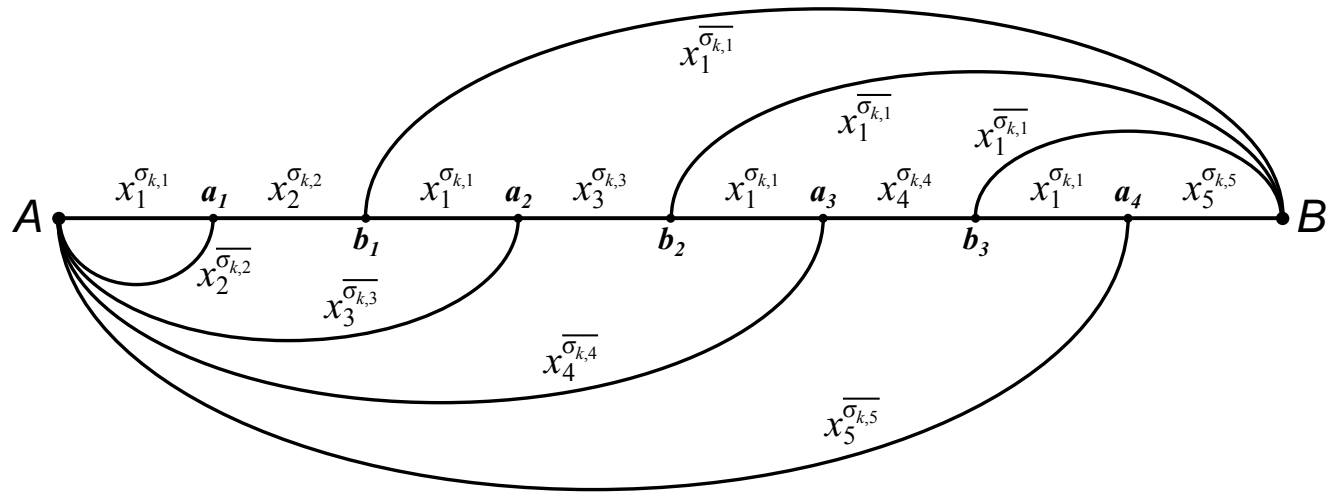

Рис. 2.

4. Неисправен контакт $x_{s+1}^{\overline{\sigma_{k, s+1}}}$, соединяющий вершины $A$ и $a_{s}$ схемы $S_{k}$ для некоторого $s \geqslant 1$. Тогда на наборе $\tilde{\pi}_{s+1, \overline{\sigma_{k, 1}}, \sigma_{k, s+1}}$ цепь $A-x_{s+1}^{\overline{\sigma_{k, s+1}}}-a_{s}-x_{s+1}^{\sigma_{k, s+1}}-b_{s}-x_{1}^{\overline{\sigma_{k, 1}}}-B$ будет проводящей и схема $S_{k}$, а значит, и $S$, выдаст единицу.

5 . Неисправен контакт $x_{s+1}^{\sigma_{k, s+1}}$, соединяющий вершины $a_{s}$ и $b_{s}$ схемы $S_{k}$ для некоторого $s \geqslant 1$. Тогда на наборе $\tilde{\pi}_{s+1, \overline{\sigma_{k, 1}}, \overline{\sigma_{k, s+1}}}$ цепь $A-x_{s+1}^{\overline{\sigma_{k, s+1}}}-a_{s}-x_{s+1}^{\sigma_{k, s+1}}-b_{s}-x_{1}^{\overline{\sigma_{k, 1}}}-B$ будет проводящей и схема $S_{k}$, а значит, и $S$, выдаст единицу.

6. Неисправен контакт $x_{n}^{\overline{\sigma_{k, n}}}$, соединяющий вершины $A$ и $a_{n-1}$ схемы $S_{k}$. Тогда на наборе $\tilde{\pi}_{n, 0, \sigma_{k, n}}$ цепь $A-x_{n}^{\overline{\sigma_{k, n}}}-a_{n-1}-x_{n}^{\sigma_{k, n}}-B$ будет проводящей и схема $S_{k}$, а значит, и $S$, выдаст единицу.

7. Неисправен контакт $x_{n}^{\sigma_{k, n}}$, соединяющий вершины $a_{n-1}$ и $B$ схемы $S_{k}$. Тогда на наборе $\tilde{\pi}_{n, 0, \overline{\sigma_{k, n}}}$ цепь $A-x_{n}^{\overline{\sigma_{k, n}}}-a_{n-1}-x_{n}^{\sigma_{k, n}}-B$ будет проводящей и схема $S_{k}$, а значит, и $S$, выдаст единицу.

Получаем, что множество $M$ является единичным проверяющим тестом для неизбыточной схемы $S$. Его длина равна $m$, поэтому $D_{s, \text { detect }}(f) \leqslant m$. Теорема 1 доказана.

Теорема 2. Справедливо неравенство $D_{s, \text { detect }}(n) \leqslant 2 n$, причём любую булеву функиию $f\left(x_{1}, \ldots, x_{n}\right)$ можно реализовать неизбыточной контактной схемой, содержащей не более одной фиктивной входной переменной и допускающей единичный проверяющий тест замыкания, длина которого не превосходит $2 n$.

Замечание 1 . Неравенство $D_{s, \text { detect }}(n) \leqslant 2 n$ следует из второго утверждения теоремы 2 .

Доказательству теоремы 2 предпошлём следующую лемму.

Лемма 1. Пусть булева функиия $f\left(x_{1}, \ldots, x_{n}\right)$, где $n \geqslant 2$, такова, что для любъх $j \in\{1, \ldots, n\}, \beta \in\{0,1\}$ существует нулевой для неё набор $\tilde{\sigma}_{j, \beta}, j$-й разряд которого равен $\beta$. Тогда для функиии $f\left(x_{1}, \ldots, x_{n}, y\right)$, фиктивно зависящей от переменной y и равной $f\left(x_{1}, \ldots, x_{n}\right)$, существует покрывающее множество $M$ из не более чем $2 n$ наборов. 
Доказательство. Множество всех наборов $\tilde{\sigma}_{j, \beta}$ при $j=1, \ldots, n, \beta=0,1$ обозначим через $A$. Наборы $\tilde{\sigma}_{1,0}$ и $\tilde{\sigma}_{1,1}$ различны, поэтому $|A| \geqslant 2$. Определим множество $A^{\prime}$ следующим образом. Пусть вначале $|A| \geqslant 3$. Возьмём произвольные три попарно различных набора из множества $A$. Очевидно, что какие-то два из них различаются по крайней мере в двух разрядах. Обозначим эти два набора через $\tilde{\delta}$ и $\tilde{\delta}^{\prime}$, а номера произвольных двух разрядов, в которых они различаются - через $j_{1}$ и $j_{2}$. Пусть $\tilde{\delta}=\left(\delta_{1}, \ldots, \delta_{n}\right)$. Положим $A^{\prime}=\{\tilde{\delta}\} \cup\left\{\tilde{\delta}^{\prime}\right\} \cup\left\{\tilde{\sigma}_{j, \overline{\delta_{j}}} \mid j \in\{1, \ldots, n\}, j \neq j_{1}, j \neq j_{2}\right\}$. Тогда $A^{\prime} \subseteq A$ и $\left|A^{\prime}\right| \leqslant n$. Если же $|A|=2$, то положим $A^{\prime}=A$, тогда $\left|A^{\prime}\right|=2 \leqslant n$. Покажем, что для любых $j \in\{1, \ldots, n\}, \beta \in\{0,1\}$ в $A^{\prime}$ найдётся набор, $j$-й разряд которого равен $\beta$ (обозначим это свойство через $\left(^{*}\right)$ ). В случае $|A|=2$ оно верно в силу равенства $A^{\prime}=A$ и определения множества $A$ и наборов $\tilde{\sigma}_{j_{2} \beta}$. Пусть $|A| \geqslant 3$. Если $j=j_{1}$ или $j=j_{2}$, то искомый набор найдётся среди наборов $\tilde{\delta}, \tilde{\delta}^{\prime}$. Пусть теперь $j \neq j_{1}$ и $j \neq j_{2}$. Если $\beta=\delta_{j}$, то в качестве искомого набора можно взять $\tilde{\delta}$. Если же $\beta=\overline{\delta_{j}}$, то в качестве искомого набора можно взять $\tilde{\sigma}_{j, \overline{\delta_{j}}}$. Таким образом, свойство $(*)$ имеет место. Далее, все наборы из множества $A^{\prime}$ обозначим через $\tilde{\delta}_{1}, \ldots, \tilde{\delta}_{k}$, $k \leqslant n$. Тогда $f\left(\tilde{\delta}_{1}\right)=\ldots=f\left(\tilde{\delta}_{k}\right)=0$ в силу включения $A^{\prime} \subseteq A$ и определения множества $A$. Через $\left(\tilde{\delta}_{s}, \alpha\right)$ будем обозначать набор длины $n+1$, получающийся из набора $\tilde{\delta}_{s}$ приписыванием к нему в конце булевой константы $\alpha$. Из определения функции $f\left(x_{1}, \ldots, x_{n}, y\right)$ следует, что $f\left(\tilde{\delta}_{s}, \alpha\right)=f\left(\tilde{\delta}_{s}\right)=0$ для $\alpha=0,1, s=1, \ldots, k$, и множество $M=\left\{\left(\tilde{\delta}_{s}, \alpha\right) \mid s=1, \ldots, k ; \alpha=0,1\right\}$ будет покрывающим для функции $f\left(x_{1}, \ldots, x_{n}, y\right)$. Действительно, возьмём произвольные числа $j \in\{1, \ldots, n\}, \alpha, \beta \in$ $\{0,1\}$. В силу свойства $\left(^{*}\right)$ в множестве $A^{\prime}$ найдётся набор $\tilde{\delta}_{s}, j$-й разряд которого равен $\beta$. Тогда набор $\left(\tilde{\delta}_{s}, \alpha\right)$ принадлежит множеству $M$, его $(n+1)$-й разряд равен $\alpha$, а $j$-й разряд - $\beta$, откуда следует, что $M-$ покрывающее множество для функции $f\left(x_{1}, \ldots, x_{n}, y\right)$ (при $\left.i=n+1\right)$. Оно содержит $2 k \leqslant 2 n$ наборов. Лемма 1 доказана.

Доказательство (теоремы 2). Пусть $f\left(x_{1}, \ldots, x_{n}\right)$ - произвольная булева функция от $n$ переменных. Без ограничения общности будем считать, что она существенно зависит от всех своих переменных. (В противном случае можно доказать теорему 2 для функции, получающейся из функции $f\left(x_{1}, \ldots, x_{n}\right)$ путём изъятия всех её фиктивных переменных, и использовать для реализации самой функции $f\left(x_{1}, \ldots, x_{n}\right)$ удовлетворяющую условиям этой теоремы контактную схему, фиктивная входная переменная которой, если она существует, отлична от всех переменных $\left.x_{1}, \ldots, x_{n}\right)$. Если $n=0$, то функция $f-$ булева константа, и её можно реализовать (неизбыточной) контактной схемой, не содержащей ни одного контакта, а в таком случае пустое множество будет единичным проверяющим тестом для этой схемы, длина которого равна $0 \leqslant 2 n$, и утверждение теоремы выполнено. Если $n=1$, то $f=x_{1}$ или $f=\overline{x_{1}}$, а каждую из этих функций можно реализовать схемой, содержащей ровно один контакт. У такой схемы есть только одна функция неисправности тождественная единица, которую можно отличить от исходной функции $f$ на одном наборе. Множество, состоящее из этого набора, будет единичным проверяющим тестом длины $1 \leqslant 2 n$ для данной схемы, и утверждение теоремы выполнено. Далее будем считать, что $n \geqslant 2$. Рассмотрим два случая.

1. Пусть для любых $j \in\{1, \ldots, n\}, \beta \in\{0,1\}$ существует нулевой для функции $f\left(x_{1}, \ldots, x_{n}\right)$ набор, $j$-й разряд которого равен $\beta$. Тогда по лемме 1 для функции 
$f\left(x_{1}, \ldots, x_{n}, y\right)$, фиктивно зависящей от переменной $y$ и равной $f\left(x_{1}, \ldots, x_{n}\right)$, существует покрывающее множество $M$ из $m \leqslant 2 n$ наборов. По теореме 1 существует такая неизбыточная контактная схема, реализующая функцию $f\left(x_{1}, \ldots, x_{n}, y\right)$, что множество $M$ для неё является единичным проверяющим тестом замыкания, а отсюда и из соотношения $|M|=m \leqslant 2 n$ следует справедливость теоремы 2 . Случай 1 разобран.

2. Отрицание случая 1: пусть для некоторых $j_{1} \in\{1, \ldots, n\}, \beta_{1} \in$ $\{0,1\}$ все двоичные наборы длины $n, j_{1}$-й разряд которых равен $\beta_{1}$, являются единичными для функции $f\left(x_{1}, \ldots, x_{n}\right)$. Тогда легко проверить справедливость тождества $f\left(x_{1}, \ldots, x_{n}\right) \equiv x_{j_{1}}^{\beta_{1}} \vee f^{\prime}\left(x_{1}, \ldots, x_{j_{1}-1}, x_{j_{1}+1}, \ldots, x_{n}\right)$, где $f^{\prime}\left(x_{1}, \ldots, x_{j_{1}-1}, x_{j_{1}+1}, \ldots, x_{n}\right)=f\left(x_{1}, \ldots, x_{j_{1}-1}, \overline{\beta_{1}}, x_{j_{1}+1}, \ldots, x_{n}\right)$. Из него следует существование такого максимального натурального числа $r$, что функция $f\left(x_{1}, \ldots, x_{n}\right)$ представима в виде

$$
f\left(x_{1}, \ldots, x_{n}\right) \equiv x_{j_{1}}^{\beta_{1}} \vee \ldots \vee x_{j_{r}}^{\beta_{r}} \vee h
$$

где $j_{1}, \ldots, j_{r}$ - попарно различные индексы от 1 до $n, \beta_{1}, \ldots, \beta_{r}$ - булевы константы, а $h$ - булева функция от всех переменных $x_{1}, \ldots, x_{n}$, кроме переменных $x_{j_{1}}, \ldots, x_{j_{r}}$. Так как функция $f$ существенно зависит от всех своих $n$ переменных, то функция $h$ существенно зависит от всех своих $n-r$ переменных и отлична от тождественной единицы. Значит, если $n-r=0$, то $h \equiv 0$. В этом случае функция $f\left(x_{1}, \ldots, x_{n}\right)$ в силу (1) представима в виде $x_{1}^{\sigma_{1}} \vee \ldots \vee x_{n}^{\sigma_{n}}$, где $\sigma_{1}, \ldots, \sigma_{n} \in\{0,1\}$. Реализуем функцию $f$ контактной схемой, представляющей собой параллельное соединение контактов $x_{1}^{\sigma_{1}}, \ldots, x_{n}^{\sigma_{n}}$. Очевидно, что у такой схемы есть только одна функция неисправности - тождественная единица. Множество $\left\{\left(\overline{\sigma_{1}}, \ldots, \overline{\sigma_{n}}\right)\right\}$ будет единичным проверяющим тестом длины $1 \leqslant 2 n$ для этой схемы, и утверждение теоремы выполнено. Далее, пусть $n-r \geqslant 1$. Заметим, что $r$ не может быть равно $n-1$ в силу (1) и максимальности числа $r$, поэтому $n-r \geqslant 2$. Переменные функции $h$ обозначим через $x_{j_{r+1}}, \ldots, x_{j_{n}}$. Рассмотрим два подслучая.

2.1. Пусть для некоторых $k \in\{1, \ldots, n-r\}, \beta \in\{0,1\}$ все двоичные наборы длины $n-r, k$-й разряд которых равен $\beta$, являются единичными для функции $h\left(x_{j_{r+1}}, \ldots, x_{j_{n}}\right)$. Тогда легко проверить справедливость тождества

$$
h\left(x_{j_{r+1}}, \ldots, x_{j_{n}}\right) \equiv x_{j_{r+k}}^{\beta} \vee h^{\prime}\left(x_{r+1}, \ldots, x_{j_{r+k-1}}, x_{j_{r+k+1}}, \ldots, x_{j_{n}}\right),
$$

где $h^{\prime}\left(x_{r+1}, \ldots, x_{j_{r+k-1}}, x_{j_{r+k+1}}, \ldots, x_{j_{n}}\right)=h\left(x_{r+1}, \ldots, x_{j_{r+k-1}}, \bar{\beta}, x_{j_{r+k+1}}, \ldots, x_{j_{n}}\right)$. Подставив (2) в правую часть (1), получим противоречие с максимальностью числа $r$. Таким образом, случай 2.1 невозможен.

2.2. Пусть для любых $k \in\{1, \ldots, n-r\}, \beta \in\{0,1\}$ существует нулевой для функции $h\left(x_{j_{r+1}}, \ldots, x_{j_{n}}\right)$ набор, $k$-й разряд которого равен $\beta$. Тогда по лемме 1 для функции $h\left(x_{j_{r+1}}, \ldots, x_{j_{n}}, y\right)$, фиктивно зависящей от переменной $y$ и равной $h\left(x_{j_{r+1}}, \ldots, x_{j_{n}}\right)$, существует покрывающее множество $M$ из $m \leqslant 2(n-r)$ наборов. По теореме 1 существует такая неизбыточная контактная схема $S$ с входными переменными $x_{j_{r+1}}, \ldots, x_{j_{n}}, y$, реализующая функцию $h\left(x_{j_{r+1}}, \ldots, x_{j_{n}}, y\right)$, что $M$ для неё является единичным проверяющим тестом. Подсоединим параллельно к схеме $S$ пучок $P$ из контактов $x_{j_{1}}^{\beta_{1}}, \ldots, x_{j_{r}}^{\beta_{r}}$. В силу (1) полученная схема (обозначим 
её $S^{\prime}$; вид этой схемы показан на рис. 3) будет реализовывать булеву функцию $f\left(x_{1}, \ldots, x_{n}, y\right)$, фиктивно зависящую от переменной $y$ и равную $f\left(x_{1}, \ldots, x_{n}\right)$. Каждый набор $\tilde{\pi}=\left(\pi_{1}, \ldots, \pi_{n-r+1}\right)$ из множества $M$ заменим набором $\tilde{\pi}^{\prime}$ длины $n+1$, у которого в $j_{s}$-м разряде при $s=1, \ldots, r$ стоит $\overline{\beta_{s}}$, при $s=r+1, \ldots, n$ стоит $\pi_{s-r}$, а в $(n+1)$-м разряде стоит $\pi_{n-r+1}$. Получим множество $M^{\prime}$, содержащее столько же наборов, сколько и $M$. Покажем, что схема $S^{\prime}$ неизбыточна и $M^{\prime}$ для неё является единичным проверяющим тестом. Из определения покрывающего множества следует, что $|M| \geqslant 1$ и, кроме того, каждый набор из множества $M$ является нулевым для функции $h\left(x_{j_{r+1}}, \ldots, x_{j_{n}}, y\right)$. Отсюда, из (1) и построения схемы $S^{\prime}$ и множества $M^{\prime}$ следует, что $\left|M^{\prime}\right| \geqslant 1$ и каждый набор из множества $M^{\prime}$ является нулевым для функции $f\left(x_{1}, \ldots, x_{n}, y\right)$. Предположим вначале, что неисправен какой-то контакт из пучка $P$. В этом случае функцией неисправности схемы $S^{\prime}$ будет тождественная единица, а её можно отличить от функции $f\left(x_{1}, \ldots, x_{n}, y\right)$ на любом наборе из множества $M^{\prime}$. Пусть теперь все контакты из пучка $P$ в схеме $S^{\prime}$ исправны. Тогда неисправным в ней может быть только какой-то контакт в подсхеме $S$. В силу построения множества $M^{\prime}$ на каждом наборе $\tilde{\pi}^{\prime}$ из этого множества функция проводимости каждого контакта из пучка $P$ равна нулю и схема $S^{\prime}$ функционирует в точности как схема $S$ на наборе $\tilde{\pi}$, из которого был получен набор $\tilde{\pi}^{\prime}$. Так как $M$ - единичный проверяющий тест для неизбыточной схемы $S$, то на наборах из множества $M^{\prime}$ можно обнаружить любую неисправность в подсхеме $S$ схемы $S^{\prime}$. Из приведённых рассуждений следует, что схема $S^{\prime}$ неизбыточна и множество $M^{\prime}$ для неё является единичным проверяющим тестом. Его длина равна $\left|M^{\prime}\right|=|M|=m \leqslant 2(n-r) \leqslant 2 n$, откуда следует справедливость теоремы 2.

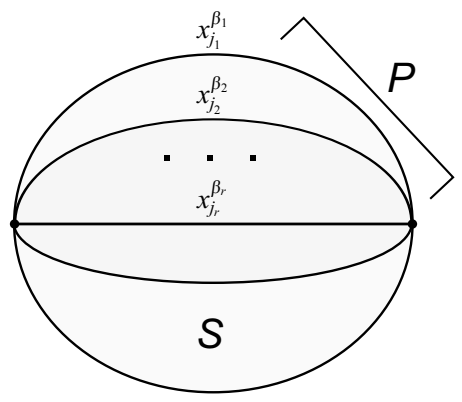

Рис. 3.

\section{3. Единичные диагностические тесты замыкания}

Теорема 3. Пусть для булевой функиии $f\left(x_{1}, \ldots, x_{n}\right)$, где $n \geqslant 3$, существуют два непересекающихся покрывающих множества $M_{1}$ и $M_{2}$ из $m_{1}$ u $m_{2}$ наборов соответственно. Тогда $D_{s, \text { diagn }}(f) \leqslant m_{1}+m_{2}$, причём существует такая неизбъточная контактная схема, реализующая функцию $f\left(x_{1}, \ldots, x_{n}\right)$, что множество $M_{1} \cup M_{2}$ для неё является единичным диагностическим тестом замыкания и любая её функиия неисправности хотя бы на одном наборе из $M_{1} \cup M_{2}$ равна нулю.

Из теоремы 3 и утверждения 1 вытекает следующее утверждение. 
Следствие 2. Для почти всех булевых функций $f$ om $n$ переменных справедливо неравенство $D_{s, \operatorname{diagn}}(f) \leqslant 8$.

Доказательство (теоремы 3). Обозначим через $I_{M_{j}}, j=1,2$, булеву функцию от $n$ переменных, равную единице на всех наборах из множества $M_{j}$ и нулю на всех остальных наборах. На каждом наборе из множества $M_{2}$ функции $f$ и $I_{M_{1}}$, а значит, и $f \vee I_{M_{1}}$, обращаются в нуль. Поэтому множество $M_{2}$ является покрывающим не только для $f$, но и для функции $f \vee I_{M_{1}}$. Аналогично множество $M_{1}$ является покрывающим для функции $f \vee I_{M_{2}}$. Пусть $S_{1}$ и $S_{2}$ - неизбыточные контактные схемы, реализующие соответственно функции $f \vee I_{M_{1}}$ и $f \vee I_{M_{2}}$, для которых множества $M_{2}$ и $M_{1}$ соответственно являются единичными проверяющими тестами; существование таких схем следует из теоремы 1 . Через $S$ обозначим схему, представляющую собой последовательное соединение схем $S_{1}$ и $S_{2}$. Тогда схема $S$ реализует функцию $\left(f \vee I_{M_{1}}\right) \&\left(f \vee I_{M_{2}}\right)=f \vee f I_{M_{1}} \vee f I_{M_{2}} \vee I_{M_{1}} I_{M_{2}}=f \vee I_{M_{1}} I_{M_{2}}=f$, так как $M_{1} \cap M_{2}=\varnothing$. Покажем, что схема $S$ неизбыточна, множество $M_{1} \cup M_{2}$ для неё является единичным проверяющим тестом и любая функция неисправности $g$ схемы $S$ равна нулю хотя бы на одном наборе из множества $M_{1} \cup M_{2}$. Пусть неисправен некоторый контакт в подсхеме $S_{1}$. Так как $M_{2}$ - единичный проверяющий тест для неизбыточной схемы $S_{1}$, то на некотором наборе $\tilde{\sigma} \in M_{2}$ значение получающейся функции неисправности $g_{1}$ схемы $S_{1}$ отличается от $\left(f \vee I_{M_{1}}\right)(\tilde{\sigma})$. Но $\left(f \vee I_{M_{1}}\right)(\tilde{\sigma})=0$ в силу того, что все наборы из $M_{2}$ - нулевые для функции $f \vee I_{M_{1}}$, поэтому $g_{1}(\tilde{\sigma})=1$. Тогда функция неисправности $g$ всей схемы $S$, равная $g_{1} \&\left(f \vee I_{M_{2}}\right)$, на наборе $\tilde{\sigma}$ принимает значение $1 \&(0 \vee 1)=1$, в то время как $f(\tilde{\sigma})=0$, т.е. функция неисправности схемы $S$ отличается от исходной функции, реализуемой данной схемой, на некотором наборе из множества $M_{1} \cup M_{2}$. С другой стороны, на любом наборе из множества $M_{1}$ функции $f$ и $I_{M_{2}}$, а значит, и функция $g=g_{1} \&\left(f \vee I_{M_{2}}\right)$, равны нулю. Аналогично рассматривается случай, когда неисправен некоторый контакт в подсхеме $S_{2}$. Получаем, что схема $S$ неизбыточна, $M_{1} \cup M_{2}$ - единичный проверяющий тест для этой схемы и любая её функция неисправности равна нулю хотя бы на одном наборе из множества $M_{1} \cup M_{2}$.

Докажем теперь, что $M_{1} \cup M_{2}$ - единичный диагностический тест для схемы $S$. Пусть $g$ - произвольная функция неисправности этой схемы, а $\tilde{\delta}-$ произвольный двоичный набор длины $n$, не принадлежащий множеству $M_{1} \cup M_{2}$. Если $f(\tilde{\delta})=1$, то $g(\tilde{\delta})=1$ в силу того, что при замыкании любого контакта в схеме $S$ реализуемая этой схемой функция может только увеличиться. Если же $f(\tilde{\delta})=0$, то и каждая из функций $f \vee I_{M_{1}}, f \vee I_{M_{2}}$ на наборе $\tilde{\delta}$ обращается в нуль, так как $\tilde{\delta} \notin M_{1} \cup M_{2}$. Это означает, что каждая из подсхем $S_{1}, S_{2}$ на наборе $\tilde{\delta}$ выдает нуль. Если неисправен какой-то контакт в подсхеме $S_{1}\left(S_{2}\right)$, то подсхема $S_{2}$ (соответственно $\left.S_{1}\right)$, а значит, и вся схема $S$, на наборе $\tilde{\delta}$ по-прежнему будет выдавать нуль, откуда следует, что любая функция неисправности схемы $S$ на этом наборе обращается в нуль. Поэтому $g(\tilde{\delta})=0$. Получаем, что функции $f$ и $g$ принимают одинаковые значения на всех наборах, не принадлежащих множеству $M_{1} \cup M_{2}$. Следовательно, любая функция неисправности схемы $S$ может отличаться от функции $f$ только на набоpax из множества $M_{1} \cup M_{2}$. Но тогда любые две различные функции неисправности схемы $S$ могут отличаться друг от друга только на наборах из этого множества и обязаны отличаться хотя бы на одном таком наборе, откуда следует, что $M_{1} \cup M_{2}-$ 
единичный диагностический тест для схемы $S$. Его длина равна $m_{1}+m_{2}$, поэтому $D_{s, \operatorname{diagn}}(f) \leqslant m_{1}+m_{2}$. Теорема 3 доказана.

Теорема 4. Справедливо неравенство $D_{s, \text { diagn }}(n) \leqslant 4 n$, причём любую булеву функиию $f\left(x_{1}, \ldots, x_{n}\right)$ можно реализовать неизбыточной контактной схемой, содержащей не более двух фиктивных входных переменных и допускающей единичный диагностический тест замыкания, длина которого не превосходит $4 n$.

Замечание 2 . Неравенство $D_{s, \text { diagn }}(n) \leqslant 4 n$ следует из второго утверждения теоремы 4.

Лемма 2. Пусть булева функиия $f\left(x_{1}, \ldots, x_{n}\right)$, где $n \geqslant 2$, такова, что для любъх $j \in\{1, \ldots, n\}, \beta \in\{0,1\}$ существует нулевой для неё набор $\tilde{\sigma}_{j, \beta}, j$-й разряд которого равен $\beta$. Тогда для функиии $f\left(x_{1}, \ldots, x_{n}, y, z\right)$, фиктивно зависящей от переменных $y, z$ и равной $f\left(x_{1}, \ldots, x_{n}\right)$, существуют два непересекающихся покрывающих множества $M_{1}$ и $M_{2}$, каждое из которьх содержит не более чем $2 n$ наборов.

Доказательство. Условия леммы 2 совпадают с условиями леммы 1. В ходе доказательства леммы 1 для функции $f\left(x_{1}, \ldots, x_{n}, y\right)$, фиктивно зависящей от переменной $y$ и равной $f\left(x_{1}, \ldots, x_{n}\right)$, было построено покрывающее множество $M=$ $\left\{\left(\tilde{\delta}_{s}, \alpha\right) \mid s=1, \ldots, k ; \alpha=0,1\right\}$ (при $i=n+1$ ), где $k \leqslant n$, все наборы $\tilde{\delta}_{1}, \ldots, \tilde{\delta}_{k}$ имеют длину $n$ и попарно различны. Из процесса построения этого множества нетрудно заметить, что $k \geqslant 2$. Через $\left(\tilde{\delta}_{s}, \alpha, \alpha^{\prime}\right)$ будем обозначать набор длины $n+2$, получающийся из набора $\left(\tilde{\delta}_{s}, \alpha\right)$ приписыванием к нему в конце булевой константы $\alpha^{\prime}$. Пусть $M_{1}=\left\{\left(\tilde{\delta}_{1}, 0,0\right)\right\} \cup\left\{\left(\tilde{\delta}_{s}, 0,1\right) \mid s=2, \ldots, k\right\} \cup\left\{\left(\tilde{\delta}_{1}, 1,0\right)\right\} \cup\left\{\left(\tilde{\delta}_{s}, 1,1\right) \mid s=2, \ldots, k\right\}$, $M_{2}=\left\{\left(\tilde{\delta}_{1}, 0,1\right)\right\} \cup\left\{\left(\tilde{\delta}_{s}, 0,0\right) \mid s=2, \ldots, k\right\} \cup\left\{\left(\tilde{\delta}_{1}, 1,1\right)\right\} \cup\left\{\left(\tilde{\delta}_{s}, 1,0\right) \mid s=2, \ldots, k\right\}$. Ясно, что множества $M_{1}$ и $M_{2}$ не пересекаются и каждое из них содержит $2 k \leqslant 2 n$ наборов. Покажем, что оба этих множества являются покрывающими для функции $f\left(x_{1}, \ldots, x_{n}, y, z\right)$. Для любого набора $\left(\tilde{\delta}_{s}, \alpha, \alpha^{\prime}\right) \in M_{1}$ имеем $f\left(\tilde{\delta}_{s}, \alpha, \alpha^{\prime}\right)=f\left(\tilde{\delta}_{s}\right)=$ $f\left(\tilde{\delta}_{s}, \alpha\right)=0$, так как $\left(\tilde{\delta}_{s}, \alpha\right) \in M$. Далее, возьмём произвольные числа $j \in\{1, \ldots, n\}$, $\alpha, \beta \in\{0,1\}$. Так как $M-$ покрывающее множество при $i=n+1$, то существует набор из $M,(n+1)$-й разряд которого равен $\alpha$, а $j$-й разряд $-\beta$. Этот набор имеет вид $\left(\tilde{\delta}_{s}, \alpha\right)$. По построению какой-то один из наборов $\left(\tilde{\delta}_{s}, \alpha, 0\right),\left(\tilde{\delta}_{s}, \alpha, 1\right)$ принадлежит множеству $M_{1} ;(n+1)$-й разряд этого набора равен $\alpha$, а $j$-й разряд - $\beta$. Наконец, для любых $\alpha, \beta \in\{0,1\}$ в $M_{1}$ по построению этого множества содержится набор, $(n+1)$-й разряд которого равен $\alpha, \mathrm{a}(n+2)$-й разряд $-\beta$. Всё вышесказанное позволяет утверждать, что множество $M_{1}$ является покрывающим для функции $f\left(x_{1}, \ldots, x_{n}, y, z\right)$. Аналогичные рассуждения можно провести и для множества $M_{2}$. Лемма 2 доказана.

Доказательство (теоремы 4). Без ограничения общности будем считать, что функция $f\left(x_{1}, \ldots, x_{n}\right)$ существенно зависит от всех своих переменных (см. комментарий в скобках в начале доказательства теоремы 2). Если $n=0$, то функция $f$ - булева константа и её можно реализовать контактной схемой, не содержащей ни одного контакта, а в таком случае пустое множество будет единичным диагностическим тестом для этой схемы, длина которого равна $0 \leqslant 4 n$, и утверждение теоремы выполнено. Если $n=1$, то $f=x_{1}$ или $f=\overline{x_{1}}$, а каждую из этих функций можно реализовать схемой, содержащей ровно один контакт. У такой схемы есть 
только одна функция неисправности - тождественная единица, которую можно отличить от исходной функции $f$ на одном наборе. Множество, состоящее из этого набора, будет единичным диагностическим тестом длины $1 \leqslant 4 n$ для данной схемы, и утверждение теоремы выполнено. Далее будем считать, что $n \geqslant 2$. Рассмотрим два случая.

1. Пусть для любых $j \in\{1, \ldots, n\}, \beta \in\{0,1\}$ существует нулевой для функции $f\left(x_{1}, \ldots, x_{n}\right)$ набор, $j$-й разряд которого равен $\beta$. Тогда по лемме 2 для функции $f\left(x_{1}, \ldots, x_{n}, y, z\right)$, фиктивно зависящей от переменных $y, z$ и равной $f\left(x_{1}, \ldots, x_{n}\right)$, существуют два не пересекающихся покрывающих множества $M_{1}$ и $M_{2}$ из $m_{1}$ и $m_{2}$ наборов соответственно, где $m_{1} \leqslant 2 n$ и $m_{2} \leqslant 2 n$. По теореме 3 существует такая неизбыточная контактная схема, реализующая функцию $f\left(x_{1}, \ldots, x_{n}, y, z\right)$, что множество $M_{1} \cup M_{2}$ для неё является единичным диагностическим тестом замыкания, а отсюда и из соотношения $\left|M_{1} \cup M_{2}\right|=m_{1}+m_{2} \leqslant 2 n+2 n=4 n$ следует справедливость теоремы 4. Случай 1 разобран.

2. Отрицание случая 1: пусть для некоторых $j_{1} \in\{1, \ldots, n\}, \beta_{1} \in\{0,1\}$ все двоичные наборы длины $n, j_{1}$-й разряд которых равен $\beta_{1}$, являются единичными для функции $f\left(x_{1}, \ldots, x_{n}\right)$. Эти условия совпадают с условиями случая 2 из доказательства теоремы 2. Дословно повторяем рассуждения из разбора указанного случая вплоть до окончания разбора случая 2.1 с заменой понятия "единичный проверяющий тест" на понятие "единичный диагностический тест" и неравенства $1 \leqslant 2 n$ на неравенство $1 \leqslant 4 n$. Получаем, что достаточно доказать теорему 4 в случае, условия которого совпадают с условиями случая 2.2 из доказательства теоремы 2. А именно, пусть для любых $k \in\{1, \ldots, n-r\}, \beta \in\{0,1\}$ существует нулевой для функции $h\left(x_{j_{r+1}}, \ldots, x_{j_{n}}\right)$ набор, $k$-й разряд которого равен $\beta$, где $h\left(x_{j_{r+1}}, \ldots, x_{j_{n}}\right)-$ функция, участвующая в правой части представления (1) для максимально возможного натурального $r$, существенно зависящая от всех своих переменных, и $n-r \geqslant 2$. Тогда по лемме 2 для функции $h\left(x_{j_{r+1}}, \ldots, x_{j_{n}}, y, z\right)$, фиктивно зависящей от переменных $y, z$ и равной $h\left(x_{j_{r+1}}, \ldots, x_{j_{n}}\right)$, существуют два непересекающихся покрывающих множества $M_{1}$ и $M_{2}$, каждое из которых содержит не более чем $2(n-r)$ наборов. По теореме 3 существует такая неизбыточная контактная схема $S$ с входными переменными $x_{j_{r+1}}, \ldots, x_{j_{n}}, y, z$, реализующая функцию $h\left(x_{j_{r+1}}, \ldots, x_{j_{n}}, y, z\right)$, что множество $M=M_{1} \cup M_{2}$ для неё является единичным диагностическим тестом и любая её функция неисправности хотя бы на одном наборе из $M$ равна нулю (последнее утверждение обозначим через $(* *))$. Подсоединим параллельно к схеме $S$ пучок $P$ из контактов $x_{j_{1}}^{\beta_{1}}, \ldots, x_{j_{r}}^{\beta_{r}}$. В силу $(1)$ полученная схема (обозначим её $S^{\prime}$ ) будет реализовывать булеву функцию $f\left(x_{1}, \ldots, x_{n}, y, z\right)$, фиктивно зависящую от переменных $y, z$ и равную $f\left(x_{1}, \ldots, x_{n}\right)$. Схема $S^{\prime}$ имеет такой же вид, что и схема $S^{\prime}$, построенная при разборе случая 2.2 в доказательстве теоремы 2 и изображенная на рис. 3, но подсхема $S$ в них устроена по-разному.

Каждый набор $\tilde{\pi}=\left(\pi_{1}, \ldots, \pi_{n-r+2}\right)$ из множества $M$ заменим набором $\tilde{\pi}^{\prime}$ длины $n+2$, у которого в $j_{s}$-м разряде при $s=1, \ldots, r$ стоит $\overline{\beta_{s}}$, при $s=r+1, \ldots, n$ стоит $\pi_{s-r}$, в $(n+1)$-м разряде стоит $\pi_{n-r+1}$, а в $(n+2)$-м разряде $-\pi_{n-r+2}$. Получим множество $M^{\prime}$, содержащее столько же наборов, сколько и $M$. Покажем, что схема $S^{\prime}$ неизбыточна и $M^{\prime}$ для неё является единичным диагностическим тестом. Из определения покрывающего множества следует, что $|M| \geqslant 1$ и, кроме того, каждый 
набор из множества $M$ является нулевым для функции $h\left(x_{j_{r+1}}, \ldots, x_{j_{n}}, y, z\right)$. Отсюда, из (1) и построения схемы $S^{\prime}$ и множества $M^{\prime}$ следует, что $\left|M^{\prime}\right| \geqslant 1$ и каждый набор из множества $M^{\prime}$ является нулевым для функции $f\left(x_{1}, \ldots, x_{n}, y, z\right)$. Пусть $g_{1}\left(x_{1}, \ldots, x_{n}, y, z\right), g_{2}\left(x_{1}, \ldots, x_{n}, y, z\right)$ - две произвольные (не обязательно различные) функции неисправности схемы $S^{\prime}$, каждая из которых возникает при неисправности какого-то контакта в подсхеме $S$. В силу построения множества $M^{\prime}$ на каждом наборе $\tilde{\pi}^{\prime}$ из этого множества функция проводимости каждого контакта из пучка $P$ равна нулю и схема $S^{\prime}$ функционирует в точности как схема $S$ на наборе $\tilde{\pi}$, из которого был получен набор $\tilde{\pi}^{\prime}$ (обозначим это утверждение через (***)). Так как $M-$ единичный диагностический тест для неизбыточной схемы $S$, то функцию $g_{1}$ можно отличить от функции $f\left(x_{1}, \ldots, x_{n}, y, z\right)$ и от $g_{2}$ (если $\left.g_{1} \not \equiv g_{2}\right)$ на наборах из множества $M^{\prime}$. Кроме того, из $(* *)$ и $(* * *)$ следует, что функция $g_{1}$ равна нулю хотя бы на одном наборе $\tilde{\sigma}$ из множества $M^{\prime}$. Предположим теперь, что все контакты в подсхеме $S$ исправны. Тогда неисправным может быть только какой-то контакт из пучка $P$. В этом случае функцией неисправности схемы $S^{\prime}$ будет тождественная единица; её можно отличить от функции $f\left(x_{1}, \ldots, x_{n}, y, z\right)$ на любом наборе из множества $M^{\prime}$, а от функции $g_{1}$ - на наборе $\tilde{\sigma} \in M^{\prime}$. Так как в качестве $g_{1}, g_{2}$ можно взять произвольные функции неисправности схемы $S^{\prime}$, каждая из которых возникает при неисправности какого-то контакта в подсхеме $S$, то из приведённых рассуждений следует, что схема $S^{\prime}$ неизбыточна и множество $M^{\prime}$ для неё является единичным диагностическим тестом. Его длина равна $\left|M^{\prime}\right|=|M|=\left|M_{1}\right|+\left|M_{2}\right| \leqslant 2(n-r)+2(n-r) \leqslant 4 n$, откуда следует справедливость теоремы 4.

\section{Список литературы}

1. Лупанов О. Б., Асимптотические ощенки сложности управляющих систем, Издательство МГУ, Москва, 1984, 138 с.

2. Чегис И. А., Яблонский С. В., “Логические способы контроля работы электрических схем", Труды МИАН, 51 (1958), 270-360.

3. Яблонский С. В., "Надёжность и контроль управляющих систем", Материаль Всесоюзного семинара по дискретной математике и её приложениям, 1986, 7-12.

4. Яблонский С. В., "Некоторые вопросы надёжности и контроля управляющих систем", Математические вопросы кибернетики, 1988, № 1, 5-25.

5. Редькин Н. П., Надёжность и диагностика схем, Издательство МГУ, Москва, 1992, $192 \mathrm{c.}$

6. Яблонский С. В., Введение в дискретную математику, Наука, Москва, 1986, 384 с.

7. Романов Д. С., "О синтезе контактных схем, допускающих короткие проверяющие тесты”, Учёные записки Казанского университета. Физико-математические науки, 156:3 (2014), 110-115.

8. Мадатян Х. А., "Полный тест для бесповторных контактных схем", Проблемы кибернетики, 1970, № 23, 103-118.

9. Редькин Н. П., "О полных проверяющих тестах для контактных схем”, Методы дискретного анализа в исследовании экстремальных структур, 39 (1983), 80-87.

10. Редькин Н. П., "О проверяющих тестах замыкания и размыкания", Методы дискретного анализа в оптимизации управляющих систем, 40 (1983), 87-99. 